\title{
Notes on the Hermitian Positive Definite Solutions of a Matrix Equation
}

\author{
Jing $\mathrm{Li}^{1}$ and Yuhai Zhang ${ }^{2}$ \\ ${ }^{1}$ School of Mathematics and Statistics, Shandong University, Weihai 264209, China \\ ${ }^{2}$ School of Mathematics, Shandong University, Jinan 250100, China \\ Correspondence should be addressed to Jing Li; xlijing@sdu.edu.cn
}

Received 15 January 2014; Revised 26 March 2014; Accepted 15 April 2014; Published 6 May 2014

Academic Editor: Alexander Timokha

Copyright (c) $2014 \mathrm{~J}$. Li and Y. Zhang. This is an open access article distributed under the Creative Commons Attribution License, which permits unrestricted use, distribution, and reproduction in any medium, provided the original work is properly cited.

\begin{abstract}
The nonlinear matrix equation, $X-\sum_{i=1}^{m} A_{i}^{*} X^{\delta_{i}} A_{i}=Q$, with $-1 \leq \delta_{i}<0$ is investigated. A fixed point theorem in partially ordered sets is proved. And then, by means of this fixed point theorem, the existence of a unique Hermitian positive definite solution for the matrix equation is derived. Some properties of the unique Hermitian positive definite solution are obtained. A residual bound of an approximate solution to the equation is evaluated. The theoretical results are illustrated by numerical examples.
\end{abstract}

\section{Introduction}

In this paper we consider the Hermitian positive definite solution of the following nonlinear matrix equation:

$$
X-\sum_{i=1}^{m} A_{i}^{*} X^{\delta_{i}} A_{i}=Q
$$

where $-1 \leq \delta_{i}<0(i=1,2, \ldots, m), A_{1}, A_{2}, \ldots, A_{m}$ are $n \times n$ complex matrices, $Q$ is an $n \times n$ Hermitian positive definite matrix, and $m$ is a positive integer. Here, $A_{i}^{*}$ denotes the conjugate transpose of the matrix $A_{i}$.

This type of nonlinear matrix equations arises in many practical applications. Equation (1) in the case $m=1$ comes from ladder networks, dynamic programming, control theory, stochastic filtering, statistics, and so forth [1-7]. When $m>1$, (1) in the case $\delta_{1}=\delta_{2}=\cdots=\delta_{m}=-1$ is recognized as playing an important role in modeling certain optimal interpolation problems (see $[8,9]$ for more details). The Hermitian positive definite solutions of the generaltype equation (1) play an important role in connection with a certain system of linear equations in many physical calculations (see $[7,10]$ for more details). When solving the nonlinear matrix equation (1), we often do not avoid some round-off errors. Then we only get an approximation $\widetilde{X}$. After we computed $\widetilde{X}$, we would like to know how good our computation was. Motivated by these, we consider in this paper the Hermitian positive definite solutions and the residual bound of (1).

In the last few years, (1) was investigated in some special cases. For the nonlinear matrix equations, $X \pm A^{*} X^{-1} A=Q$ [11-17], $X+A^{*} X^{-1} A+B^{*} X^{-1} B=Q[18], X-A^{*} X^{-2} A=Q$ $[19,20], X^{s}-A^{*} X^{-t} A=Q[21], X-A^{*} X^{-q} A=Q[22-$ 25], and $X-\sum_{i=1}^{m} A_{i}^{*} X^{-1} A_{i}=Q[9,26,27]$, there were many contributions in the literature to the solvability, numerical solutions, and perturbation analysis. In addition, the related general equations, $X \pm A^{*} \mathscr{F}(X) A=Q$ [28-34], were studied by some scholars.

For the case $m>1$ and $\delta_{i}$ changes with $i$, Duan et al. [35] proved that (1) with $0<\left|\delta_{i}\right|<1$ has a unique Hermitian positive definite solution by fixed point theorems for monotone and mixed monotone operators in a normal cone. Lim [36] showed that (1) with $0<\left|\delta_{i}\right|<1$ has a unique Hermitian positive definite solution by using a strict contraction for the Thompson metric on the open convex cone of positive definite matrices. Shi et al. [37] studied the existence and uniqueness of solutions of nonlinear matrix equations, $X=\sum_{l=0}^{k-1} P_{l}^{T} X^{\alpha_{l}} P_{l}$ and $X=\sum_{l=0}^{k-1}\left(P_{l}^{T} X P_{l}\right)^{\alpha_{l}}$, with $\left|\alpha_{l}\right|<1$. Li [10] gave perturbation analysis for the positive definite solution of (1) with $0<\delta_{i}<1$. Duan et al. [38] gave two perturbation estimates for the positive definite solution of (1) with $0<\left|\delta_{i}\right|<1$. However, these 
papers have not considered (1) in the case $\delta_{i}=-1$ for some $i$ and the approaches in these papers will become invalid in this case. Meanwhile, in some practical problems (e.g., in certain optimal interpolation problems), the case of $\delta_{i}=$ -1 for some $i$ is required. To our best knowledge, there has been no literature paying attention to the Hermitian positive definite solutions and the residual bound for (1) with $-1 \leq \delta_{i}<0$. By using the integral representation of matrix function and the fixed point theorem, we prove the existence of a unique Hermitian positive definite solution to (1) and consider the residual bound of this equation. Note that the integral representation of matrix function $X^{\delta_{i}}$ in the case $-1<\delta_{i}<0$ is different from the case $0<\delta_{i}<1$. Furthermore, the monotonicity of $X^{\delta_{i}}$ in the former case differs from the latter. Based on the above arguments, we will consider (1) with $-1 \leq \delta_{i}<0$ in this paper.

The rest of the paper is organized as follows. In Section 2, we give some preliminary lemmas that will be needed to develop this work. In Section 3, a fixed point theorem in partially ordered sets is proved. And then, by means of this fixed point theorem, the existence of a unique Hermitian positive definite solution for the matrix equation (1) with $-1 \leq$ $\delta_{i}<0$ is derived. We propose an iterative method to compute the Hermitian positive definite solution. We also obtain some properties of the unique Hermitian positive definite solution. Furthermore, in Section 4, a residual bound for the unique Hermitian positive definite solution to (1) with $-1 \leq \delta_{i}<0$ is given. Finally, several numerical examples are presented in Section 5 .

We denote by $\mathscr{C}^{n \times n}$ the set of $n \times n$ complex matrices, by $\mathscr{H}^{n \times n}$ the set of $n \times n$ Hermitian matrices, by $I$ the identity matrix, by $\|\cdot\|$ the spectral norm, and by $\lambda_{\max }(M)$ and $\lambda_{\text {min }}(M)$ the maximal and minimal eigenvalues of $M$, respectively. For $X, Y \in \mathscr{H}^{n \times n}$, we write $X \geq Y$ (resp., $X>Y$ ) if $X-Y$ is Hermitian positive semidefinite (resp., definite). Further, the sets $[A, B]$ and $(A, B)$ are defined by $[A, B]=\{C \mid$ $A \leq C \leq B\}$ and $(A, B)=\{C \mid A<C<B\}$, respectively.

\section{Preliminaries}

Lemma 1 (see [39]). If $A \geq B>0$, then $0<A^{-1} \leq B^{-1}$.

Lemma 2 (see [39]). If $A \geq B>0$ and $0 \leq \gamma \leq 1$, then $A^{\gamma} \geq B^{\gamma}$.

For the sake of completeness we will provide the proof of the next lemma.

Lemma 3 (see [17]). For every positive definite matrix $X$, if $X+\Delta X \geq(1 / \nu) X$, with $v>0$, then

$$
\begin{aligned}
& \left\|X^{-1 / 2} A^{*}\left((X+\Delta X)^{-1}-X^{-1}\right) A X^{-1 / 2}\right\| \\
& \leq\left(\left\|X^{-1 / 2} \Delta X X^{-1 / 2}\right\|+v\left\|X^{-1 / 2} \Delta X X^{-1 / 2}\right\|^{2}\right) \\
& \quad \times\left\|X^{-1 / 2} A X^{-1 / 2}\right\|^{2} .
\end{aligned}
$$

Proof. Suppose that $X$ is a positive definite matrix. If $X+\Delta X \geq$ $(1 / \nu) I>0$, then

$$
\begin{aligned}
\left\|X^{-1 / 2} A^{*}\left((X+\Delta X)^{-1}-X^{-1}\right) A X^{-1 / 2}\right\| & \left\|X^{-1 / 2} A^{*} X^{-1} \Delta X(X+\Delta X)^{-1} A X^{-1 / 2}\right\| \\
= & \| X^{-1 / 2} A^{*} X^{-1} \Delta X X^{-1} A X^{-1 / 2} \\
& +X^{-1 / 2} A^{*} X^{-1} \Delta X X^{-1} \Delta X(X+\Delta X)^{-1} A X^{-1 / 2} \| \\
\leq & \left\|X^{-1 / 2} A^{*} X^{-1} A X^{-1 / 2}\right\|\left\|X^{-1 / 2} \Delta X X^{-1 / 2}\right\| \\
& +\left\|X^{-1 / 2} A X^{-1 / 2}\right\|^{2}\left\|X^{1 / 2}(X+\Delta X)^{-1} X^{1 / 2}\right\| \\
& \times\left\|X^{-1 / 2} \Delta X X^{-1 / 2}\right\|^{2} \\
\leq & \left(\left\|X^{-1 / 2} \Delta X X^{-1 / 2}\right\|+\nu\left\|X^{-1 / 2} \Delta X X^{-1 / 2}\right\|^{2}\right) \\
& \times\left\|X^{-1 / 2} A X^{-1 / 2}\right\|^{2} \cdot
\end{aligned}
$$

Lemma 4 (see [23]). For every Hermitian positive definite matrix $X$ and $\Delta X \in \mathscr{H}^{n \times n}$, it yields that

(i) $X^{-p}=(\sin p \pi / \pi) \int_{0}^{\infty}(\lambda I+X)^{-1} \lambda^{-p} d \lambda, 0<p<1$;

(ii) $X^{-p}=(\sin p \pi / p \pi) \int_{0}^{\infty}(\lambda I+X)^{-1} X(\lambda I+X)^{-1} \lambda^{-p} d \lambda$, $0<p<1$.

In addition, if $0<p<1$ and $X+\Delta X \geq(1 / \nu) X$, with $v>0$, then

$$
\begin{aligned}
& \left\|X^{-1 / 2} A^{*}\left((X+\Delta X)^{-p}-X^{-p}\right) A X^{-1 / 2}\right\| \\
& \leq p\left(\left\|X^{-1 / 2} \Delta X X^{-1 / 2}\right\|+\nu\left\|X^{-1 / 2} \Delta X X^{-1 / 2}\right\|^{2}\right) \\
& \quad \times\left\|X^{-p / 2} A X^{-1 / 2}\right\|^{2} .
\end{aligned}
$$

\section{The Positive Definite Solutions}

In this section, we use a new method, which is different from the approaches applied in $[35,36]$ to prove that (1) with $-1 \leq$ $\delta_{i}<0$ has a unique Hermitian positive definite solution $X$. Meanwhile, we give an iterative method to compute the unique Hermitian positive definite solution for arbitrary initial positive definite matrix. Moreover, we obtain some properties of the Hermitian positive definite solution to (1).

Theorem 5. Let $\Theta$ be a partially ordered metric space, with the property that, for any two elements $X$ and $Y$ in $\Theta$, there is a positive number $t$ such that $Y \leq X$.

Let $\mathscr{F}: \Theta \rightarrow \Theta$ be a continuous, order reversing map such that there is a $Q \in \Theta$ with $\mathscr{F}(X) \geq Q$ for all $X \in \Theta$. Put $S=\{X \in \Theta \mid Q \leq X \leq \mathscr{F}(Q)\}$. Then $\mathscr{F}$ maps $S$ into itself.

Consider $\mathscr{F}^{2}(X)=\mathscr{F}(\mathscr{F}(X))$. Assume in addition that for $0<t<1$ there is a number $\eta(t)>0$ such that, for all $X \in S$,

$$
\mathscr{F}^{2}(t X) \geq t(1+\eta(t)) \mathscr{F}^{2}(X) .
$$


Then $\mathscr{F}$ has a unique fixed point in $\Theta$, and, for every $X_{0} \in \Theta$, the iteration $X_{n+1}=\mathscr{F}\left(X_{n}\right)$, started with $X_{0}$, converges to the unique fixed point.

Proof. To show that $\mathscr{F}$ maps $S$ into itself, we only need to show that for $X \in S$ we have $\mathscr{F}(X) \leq \mathscr{F}(Q)$. In fact, since $\mathscr{F}$ is order reversing, something much stronger holds; for $X \geq Q$ we have $\mathscr{F}(X) \leq \mathscr{F}(Q)$. In particular, $\mathscr{F}$ maps $S$ into $S$. Moreover, $\mathscr{F}^{2}$ maps $\Theta$ into $S$. It follows that if there is a fixed point of $\mathscr{F}$, then it is in $S$.

The fact that $\mathscr{F}$ is order reversing means that $\mathscr{F}^{2}$ is order preserving and one can check that there are two matrices, $X^{(1)}$ and $X^{(2)}$, such that these two form a periodic orbit which is the attractor of the iteration of $\mathscr{F}$ for any starting value. In addition, we have $X^{(1)}=\lim _{k \rightarrow \infty} \mathscr{F}^{2 k+1}(Q)$ and $X^{(2)}=$ $\lim _{k \rightarrow \infty} \mathscr{F}^{2 k}(Q)$, so that $X^{(2)} \leq X^{(1)}$.

It remains to show that, under the extra condition (5), $X^{(1)}$ and $X^{(2)}$ are equal. In fact, we will show that $X^{(1)} \leq X^{(2)}$, which is enough. By the assumption on $\Theta$, there is a $t$ such that $t X^{(1)} \leq X^{(2)}$. Let $t_{0}=\max \left\{t \mid t X^{(1)} \leq X^{(2)}\right\}$. Suppose that $t_{0}<1$. Then, using (5),

$$
\begin{aligned}
X^{(2)} & =\mathscr{F}^{2}\left(X^{(2)}\right) \geq \mathscr{F}^{2}\left(t_{0} X^{(1)}\right) \geq t_{0}\left(1+\eta\left(t_{0}\right)\right) \mathscr{F}^{2}\left(X^{(1)}\right) \\
& >t_{0} \mathscr{F}^{2}\left(X^{(1)}\right)=t_{0} X^{(1)},
\end{aligned}
$$

which contradicts the definition of $t_{0}$. So $t_{0} \geq 1$, and in particular $X^{(1)} \leq X^{(2)}$.

The following results are immediate consequences of Theorem 5 .

Theorem 6. There exists a unique Hermitian positive definite solution $X$ of (1) with $-1 \leq \delta_{i}<0$, and the iteration,

$$
X_{0}>0, \quad X_{n}=Q+\sum_{i=1}^{m} A_{i}^{*} X_{n-1}^{\delta_{i}} A_{i}, \quad n=1,2, \ldots,
$$

converges to $X$.

Proof. Let $\mathscr{X}=\left\{X \in \mathscr{C}^{n \times n} \mid X>0\right\}$. Then the set $\mathscr{X}$ is partially ordered and for any two elements, $X$ and $Y$, in $\mathscr{X}$ there is a positive number $t$ such that $t Y \leq X$. A map $F$ associated with (1) is defined by

$$
F(X)=Q+\sum_{i=1}^{m} A_{i}^{*} X^{\delta_{i}} A_{i}, \quad X \in \mathscr{X} .
$$

Obviously, $F: \mathscr{X} \rightarrow \mathscr{X}$ is continuous, and a solution of (1) is a fixed point of $F$. Let $\Omega=[Q, F(Q)]$ and $\mathscr{L}_{i}(X)=X^{\delta_{i}}(X \in$ $\left.\Omega,-1 \leq \delta_{i}<0\right)$. By Lemmas 1 and 2 , we obtain that $\mathscr{L}_{i}$ is order reversing. So $\mathscr{L}_{i}(X) \leq \mathscr{L}_{i}(Q)$. Then

$$
\begin{aligned}
F(X) & =Q+\sum_{i=1}^{m} A_{i}^{*} \mathscr{L}_{i}(X) A_{i} \\
& \leq Q+\sum_{i=1}^{m} A_{i}^{*} \mathscr{L}_{i}(Q) A_{i}=F(Q),
\end{aligned}
$$

and as $\mathscr{L}_{i}(X)>0$ also $F(X)=Q+\sum_{i=1}^{m} A_{i}^{*} \mathscr{L}_{i}(X) A_{i} \geq Q$. That is, $F$ maps $\Omega$ into itself and $F$ is also order reversing.

By Theorem 5, it remains to prove that, for $0<t<1$, there exists a number $\eta(t)>0$ such that, for all $X \in \Omega$,

$$
F^{2}(t X) \geq t(1+\eta(t)) F^{2}(X) .
$$

In fact, choose $\eta(t)=(1-t) \lambda_{\min }(Q) / t \lambda_{\max }(F(Q))$. Note that

$$
F^{2}(X) \leq F(Q) \leq \lambda_{\max }(F(Q)) I .
$$

For $0<t<1$, a calculation gives

$$
\begin{aligned}
F^{2}( & t X)-t(1+\eta(t)) F^{2}(X) \\
= & (1-t) Q+\sum_{i=1}^{m} t^{\delta_{i}^{2}} A_{i}^{*}\left(t^{-\delta_{i}} Q+\sum_{i=1}^{m} A_{i}^{*} X^{\delta_{i}} A_{i}\right)^{\delta_{i}} A_{i} \\
& -t \sum_{i=1}^{m} A_{i}^{*}\left(Q+\sum_{i=1}^{m} A_{i}^{*} X^{\delta_{i}} A_{i}\right)^{\delta_{i}} A_{i} \\
& -t \cdot \frac{(1-t) \lambda_{\min }(Q)}{t \lambda_{\max }\left(Q+\sum_{i=1}^{m} A_{i}^{*} Q^{\delta_{i}} A_{i}\right)} F^{2}(X) \\
\geq & (1-t) \lambda_{\min }(Q) I-\frac{(1-t) \lambda_{\min }(Q) \lambda_{\max }(F(Q)) I}{\lambda_{\max }(F(Q))} \\
= & 0,
\end{aligned}
$$

which completes the proof.

Theorem 7. If $X$ is an Hermitian positive definite solution of (1), then $\beta I \leq X \leq \alpha I$, where $\alpha$ and $\beta$ are, respectively, the solutions of the following equations:

$$
\begin{aligned}
x=\lambda_{\max }(Q)+\sum_{i=1}^{m} & \lambda_{\max }\left(A_{i}^{*} A_{i}\right) \\
& \times\left(\lambda_{\min }(Q)+\sum_{i=1}^{m} \lambda_{\min }\left(A_{i}^{*} A_{i}\right) x^{\delta_{i}}\right)^{\delta_{i}},
\end{aligned}
$$

$$
\begin{aligned}
x=\lambda_{\min }(Q)+\sum_{i=1}^{m} \lambda_{\min }\left(A_{i}^{*} A_{i}\right) & \\
& \times\left(\lambda_{\max }(Q)+\sum_{i=1}^{m} \lambda_{\max }\left(A_{i}^{*} A_{i}\right) x^{\delta_{i}}\right)^{\delta_{i}} .
\end{aligned}
$$

Moreover, 
Proof. Define the sequences $\left\{\beta_{n}\right\}$ and $\left\{\alpha_{n}\right\}$ as

$$
\begin{gathered}
\beta_{0}=\lambda_{\min }(Q), \quad \beta_{n+1}=\lambda_{\min }(Q)+\sum_{i=1}^{m} \lambda_{\min }\left(A_{i}^{*} A_{i}\right) \alpha_{n}^{\delta_{i}}, \\
\alpha_{n}=\lambda_{\max }(Q)+\sum_{i=1}^{m} \lambda_{\max }\left(A_{i}^{*} A_{i}\right) \beta_{n}^{\delta_{i}}, \quad n=0,1,2, \ldots
\end{gathered}
$$

From (16), it follows that

$$
\begin{aligned}
& \lambda_{\text {min }}(Q)=\beta_{0} \leq \alpha_{0}=\lambda_{\text {max }}(Q)+\sum_{i=1}^{m} \lambda_{\text {max }}\left(A_{i}^{*} A_{i}\right) \lambda_{\text {min }}^{\delta_{i}}(Q), \\
& \lambda_{\text {min }}(Q)=\beta_{0} \leq \beta_{1}=\lambda_{\min }(Q)+\sum_{i=1}^{m} \lambda_{\min }\left(A_{i}^{*} A_{i}\right) \alpha_{0}^{\delta_{i}} \\
& \leq \lambda_{\max }(Q)+\sum_{i=1}^{m} \lambda_{\max }\left(A_{i}^{*} A_{i}\right) \lambda_{\min }^{\delta_{i}}(Q) \text {, } \\
& \lambda_{\min }(Q)=\beta_{0} \leq \alpha_{1}=\lambda_{\max }(Q)+\sum_{i=1}^{m} \lambda_{\max }\left(A_{i}^{*} A_{i}\right) \beta_{1}^{\delta_{i}} \\
& \leq \lambda_{\max }(Q)+\sum_{i=1}^{m} \lambda_{\max }\left(A_{i}^{*} A_{i}\right) \lambda_{\text {min }}^{\delta_{i}}(Q)=\alpha_{0} .
\end{aligned}
$$

Supposing $\lambda_{\min }(Q) \leq \beta_{k-1} \leq \beta_{k} \leq \lambda_{\max }(Q)+$ $\sum_{i=1}^{m} \lambda_{\max }\left(A_{i}^{*} A_{i}\right) \lambda_{\text {min }}^{\delta_{i}}(Q)$ and $\lambda_{\text {min }}(Q) \leq \alpha_{k} \leq \alpha_{k-1}$, then

$$
\begin{aligned}
\lambda_{\text {min }}(Q) & \leq \beta_{k}=\lambda_{\text {min }}(Q)+\sum_{i=1}^{m} \lambda_{\text {min }}\left(A_{i}^{*} A_{i}\right) \alpha_{k-1}^{\delta_{i}} \\
& \leq \lambda_{\text {min }}(Q)+\sum_{i=1}^{m} \lambda_{\text {min }}\left(A_{i}^{*} A_{i}\right) \alpha_{k}^{\delta_{i}} \\
& =\beta_{k+1} \leq \lambda_{\max }(Q)+\sum_{i=1}^{m} \lambda_{\max }\left(A_{i}^{*} A_{i}\right) \lambda_{\min }^{\delta_{i}}(Q), \\
\lambda_{\text {min }}(Q) & \leq \alpha_{k+1}=\lambda_{\max }(Q)+\sum_{i=1}^{m} \lambda_{\max }\left(A_{i}^{*} A_{i}\right) \beta_{k+1}^{\delta_{i}} \\
& \leq \lambda_{\max }(Q)+\sum_{i=1}^{m} \lambda_{\max }\left(A_{i}^{*} A_{i}\right) \beta_{k}^{\delta_{i}}=\alpha_{k} .
\end{aligned}
$$

Hence, for each $k$, we have $\lambda_{\text {min }}(Q) \leq \alpha_{k+1} \leq \alpha_{k}$ and $\lambda_{\text {min }}(Q) \leq \beta_{k} \leq \beta_{k+1} \leq \lambda_{\text {max }}(Q)+\sum_{i=1}^{m} \lambda_{\text {max }}\left(A_{i}^{*} A_{i}\right) \lambda_{\text {min }}^{\delta_{i}}(Q)$, which imply that the sequences $\left\{\alpha_{n}\right\}$ and $\left\{\beta_{n}\right\}$ are monotonic and bounded. Therefore, they are convergent to certain positive numbers. Let

$$
\alpha=\lim _{n \rightarrow \infty} \alpha_{n}, \quad \beta=\lim _{n \rightarrow \infty} \beta_{n} .
$$

Taking limits in (16) yields

$$
\begin{aligned}
& \alpha=\lambda_{\text {max }}(Q)+\sum_{i=1}^{m} \lambda_{\text {max }}\left(A_{i}^{*} A_{i}\right) \beta^{\delta_{i}}, \\
& \beta=\lambda_{\text {min }}(Q)+\sum_{i=1}^{m} \lambda_{\text {min }}\left(A_{i}^{*} A_{i}\right) \alpha^{\delta_{i}},
\end{aligned}
$$

which imply that

$$
\begin{aligned}
& \alpha=\lambda_{\max }(Q)+\sum_{i=1}^{m} \lambda_{\max }\left(A_{i}^{*} A_{i}\right) \\
& \times\left(\lambda_{\min }(Q)+\sum_{i=1}^{m} \lambda_{\min }\left(A_{i}^{*} A_{i}\right) \alpha^{\delta_{i}}\right)^{\delta_{i}}, \\
& \beta=\lambda_{\min }(Q)+\sum_{i=1}^{m} \lambda_{\min }\left(A_{i}^{*} A_{i}\right) \\
& \times\left(\lambda_{\max }(Q)+\sum_{i=1}^{m} \lambda_{\max }\left(A_{i}^{*} A_{i}\right) \beta^{\delta_{i}}\right)^{\delta_{i}} .
\end{aligned}
$$

Therefore $\alpha$ and $\beta$ satisfy (13) and (14), respectively. We will prove that $X \in[\beta I, \alpha I]$ for any positive definite solution $X$. According to Lemmas 1 and 2 and the sequences defined by (16), it follows that $\beta_{0} I=\lambda_{\text {min }}(Q) \leq X \leq\left(\lambda_{\max }(Q)+\right.$ $\left.\sum_{i=1}^{m} \lambda_{\max }\left(A_{i}^{*} A_{i}\right) \lambda_{\text {min }}^{\delta_{i}}(Q)\right) I=\alpha_{0} I$ for each Hermitian positive definite solution $X$. From $X=Q+\sum_{i=1}^{m} A_{i}^{*} X^{\delta_{i}} A_{i}$, it follows that $X=Q+\sum_{i=1}^{m} A_{i}^{*}\left(Q+\sum_{i=1}^{m} A_{i}^{*} X^{\delta_{i}} A_{i}\right)^{\delta_{i}} A_{i}$. Hence

$$
\begin{aligned}
\left(\lambda_{\min }(Q)+\sum_{i=1}^{m} \lambda_{\min }\right. & \left(A_{i}^{*} A_{i}\right) \\
& \times\left(\lambda_{\max }(Q)\right. \\
& \left.\left.+\sum_{i=1}^{m} \lambda_{\max }\left(A_{i}^{*} A_{i}\right) \lambda_{\min }^{\delta_{i}}(X)\right)^{\delta_{i}}\right) I
\end{aligned}
$$

$\leq X$

$$
\leq\left(\lambda_{\max }(Q)+\sum_{i=1}^{m} \lambda_{\max }\left(A_{i}^{*} A_{i}\right)\right.
$$

$$
\begin{aligned}
& \times\left(\lambda_{\min }(Q)\right. \\
& \left.\left.\quad+\sum_{i=1}^{m} \lambda_{\min }\left(A_{i}^{*} A_{i}\right) \lambda_{\max }^{\delta_{i}}(X)\right)^{\delta_{i}}\right)
\end{aligned}
$$

$\times I$. 
Since $\beta_{0} I \leq X \leq \alpha_{0} I$, it follows that $\beta_{0} \leq \lambda_{\text {min }}(X)$ and $\lambda_{\max }(X) \leq \alpha_{0}$. Note that inequality (22) implies $\beta_{1} I \leq X \leq$ $\alpha_{1} I$. By similar induction, it yields that

$$
\beta_{n} I \leq X \leq \alpha_{n} I .
$$

Taking limits on both sides of inequality (23), we have $\beta I \leq$ $X \leq \alpha I$.

Corollary 8. Every Hermitian positive definite solution of (1) is in $\left[Q+\sum_{i=1}^{m} \alpha^{\delta_{i}} A_{i}^{*} A_{i}, Q+\sum_{i=1}^{m} \beta^{\delta_{i}} A_{i}^{*} A_{i}\right]$, where $\alpha$ and $\beta$ are defined as in Theorem 7 .

Proof. We suppose that $X$ is the Hermitian positive definite solution of (1). By Theorem 7, it follows that

$$
\beta \leq \lambda_{\text {min }}(X), \quad \lambda_{\max }(X) \leq \alpha .
$$

Using $X=Q+\sum_{i=1}^{m} A_{i}^{*} X^{\delta_{i}} A_{i}$, we obtain $Q+\sum_{i=1}^{m} \lambda_{\text {max }}^{\delta_{i}}(X) A_{i}^{*} A_{i} \leq X \leq Q+\sum_{i=1}^{m} \lambda_{\text {min }}^{\delta_{i}}(X) A_{i}^{*} A_{i}$. Applying inequality (24) yields $Q+\sum_{i=1}^{m} \alpha^{\delta_{i}} A_{i}^{*} A_{i} \leq X \leq$ $Q+\sum_{i=1}^{m} \beta^{\delta_{i}} A_{i}^{*} A_{i}$.

Remark 9. The above estimate of Hermitian positive definite solution of (1) is more precise than that in Theorem 7.

\section{Residual Bound}

The matrix equation (1) with $-1 \leq \delta_{i}<0$ includes the following cases: first, some $\delta_{i}$ in $(-1,0)$ and others $\delta_{i}=-1$; secondly, all $\delta_{i}$ in $(-1,0)$; thirdly, all $\delta_{i}=-1$. Without loss of generality, let $\delta_{i}=-1, i=1,2, \ldots, l$, and $-1<\delta_{i}<0$, $i=l+1, \ldots, m$. Then (1) can be rewritten as

$$
X-\sum_{i=1}^{l} A_{i}^{*} X^{-1} A_{i}-\sum_{i=l+1}^{m} A_{i}^{*} X^{\delta_{i}} A_{i}=Q .
$$

In this section, a residual bound of an approximate solution for the unique solution to (25) is developed.

Theorem 10. Let $\widetilde{X}>0$ be an approximation to the solution $X$ of (25). If

$$
\Sigma=\sum_{i=1}^{l}\left\|\widetilde{X}^{-1 / 2} A_{i} \widetilde{X}^{-1 / 2}\right\|^{2}-\sum_{i=l+1}^{m} \delta_{i}\left\|\widetilde{X}^{\delta_{i} / 2} A_{i} \widetilde{X}^{-1 / 2}\right\|^{2}<1
$$

and the residual $R(\widetilde{X}) \equiv Q+\sum_{i=1}^{l} A_{i}^{*} \widetilde{X}^{-1} A_{i}+\sum_{i=l+1}^{m} A_{i}^{*} \widetilde{X}^{\delta_{i}} A_{i}-$ $\widetilde{X}$ satisfies

$$
\|R(\widetilde{X})\|<\frac{\theta_{1}}{2\left\|\widetilde{X}^{-1}\right\|} \min \left\{1, \frac{\theta_{1}}{2}\right\}
$$

$$
\text { where } \theta_{1} \equiv 1+\left\|\widetilde{X}^{-1}\right\|\|R(\widetilde{X})\|-\Sigma>0
$$

then

$$
\text { where } \theta=\frac{\|X-\widetilde{X}\| \leq \theta\|R(\widetilde{X})\|,}{\theta_{1}+\sqrt{\theta_{1}^{2}-4\|\widetilde{X}\|\left\|\widetilde{X}^{-1}\right\|}} \text {. }
$$

Proof. Let

$$
\Psi=\left\{\Delta X \in \mathscr{H}^{n \times n}:\left\|\widetilde{X}^{-1 / 2} \Delta X \widetilde{X}^{-1 / 2}\right\| \leq \theta_{2}\|R(\widetilde{X})\|\right\},
$$

where $\theta_{2}=\theta /\|\widetilde{X}\|$. Obviously, $\Psi$ is a nonempty bounded convex closed set. Let

$$
\begin{aligned}
g(\Delta X)= & \sum_{i=1}^{l} A_{i}^{*}\left[(\widetilde{X}+\Delta X)^{-1}-\widetilde{X}^{-1}\right] A_{i} \\
& +\sum_{i=l+1}^{m} A_{i}^{*}\left[(\widetilde{X}+\Delta X)^{\delta_{i}}-\widetilde{X}^{\delta_{i}}\right] A_{i}+R(\widetilde{X}) .
\end{aligned}
$$

Evidently $g: \Psi \mapsto \mathscr{H}^{n \times n}$ is continuous. We will prove that $g(\Psi) \subseteq \Psi$. For every $\Delta X \in \Psi$, we have

$$
\left\|\widetilde{X}^{-1 / 2} \Delta X \widetilde{X}^{-1 / 2}\right\| \leq \theta_{2}\|R(\widetilde{X})\| .
$$

Hence

$$
\widetilde{X}^{-1 / 2} \Delta X \widetilde{X}^{-1 / 2} \geq-\theta_{2}\|R(\widetilde{X})\| I .
$$

That is,

$$
\widetilde{X}+\Delta X \geq\left(1-\theta_{2}\|R(\widetilde{X})\|\right) \widetilde{X} .
$$

Using (27), one sees that

$$
\begin{aligned}
\theta_{2}\|R(\widetilde{X})\| & =\frac{2\left\|\widetilde{X}^{-1}\right\|\|R(\widetilde{X})\|}{\theta_{1}+\sqrt{\theta_{1}^{2}-4\left\|\widetilde{X}^{-1}\right\|\|R(\widetilde{X})\|}} \\
& <\frac{2\left\|\widetilde{X}^{-1}\right\|\|R(\widetilde{X})\|}{\theta_{1}}<1,
\end{aligned}
$$

which means that $\left(1-\theta_{2}\|R(\widetilde{X})\|\right) \widetilde{X}>0$.

According to Lemmas 3 and 4 and inequality (33), we obtain

$$
\begin{aligned}
& \left\|\widetilde{X}^{-1 / 2} g(\Delta X) \widetilde{X}^{-1 / 2}\right\| \\
& =\| \widetilde{X}^{-1 / 2}\left[\sum_{i=1}^{l} A_{i}^{*}\left((\widetilde{X}+\Delta X)^{-1}-\widetilde{X}^{-1}\right) A_{i}\right. \\
& \left.\quad+\sum_{i=l+1}^{m} A_{i}^{*}\left((\widetilde{X}+\Delta X)^{\delta_{i}}-\widetilde{X}^{\delta_{i}}\right) A_{i}\right] \widetilde{X}^{-1 / 2} \\
& +\widetilde{X}^{-1 / 2} R(\widetilde{X}) \widetilde{X}^{-1 / 2} \| \\
& \leq\left(\left\|\widetilde{X}^{-1 / 2} \Delta X \widetilde{X}^{-1 / 2}\right\|+\frac{\left\|\widetilde{X}^{-1 / 2} \Delta X \widetilde{X}^{-1 / 2}\right\|^{2}}{1-\theta_{2}\|R(\widetilde{X})\|}\right) \Sigma \\
& +\left\|\widetilde{X}^{-1 / 2} R(\widetilde{X}) \widetilde{X}^{-1 / 2}\right\| \\
& \leq\left(\theta_{2}\|R(\widetilde{X})\|+\frac{\left(\theta_{2}\|R(\widetilde{X})\|\right)^{2}}{1-\theta_{2}\|R(\widetilde{X})\|}\right) \Sigma \\
& +\left\|\widetilde{X}^{-1}\right\|\|R(\widetilde{X})\|=\theta_{2}\|R(\widetilde{X})\| .
\end{aligned}
$$


TABLE 1: Results for Example 2 with different values of $k$.

\begin{tabular}{ccccc}
\hline \multicolumn{1}{c||}{$k$} & 4 & 5 & 6 & 7 \\
\hline$\left.\left\|\widetilde{X}_{k}-X\right\| \widetilde{X}_{k}\right)$ & $1.2683 \times 10^{-5}$ & $7.4165 \times 10^{-7}$ & $4.3291 \times 10^{-8}$ & $2.6045 \times 10^{-9}$ \\
$\theta \| R\left(\widetilde{X}_{k}\right.$ & $1.6364 \times 10^{-5}$ & $9.5679 \times 10^{-7}$ & $5.5944 \times 10^{-8}$ & $3.2711 \times 10^{-9}$ \\
\hline
\end{tabular}

By Brouwer's fixed point theorem, there exists a $\Delta X \in \Psi$ such that $g(\Delta X)=\Delta X$. Hence $\widetilde{X}+\Delta X$ is a solution of (25). Moreover, by Theorem 6, we know that the solution $X$ of (25) is unique. Then

$$
\begin{gathered}
\|\widetilde{X}-X\|=\|\Delta X\| \leq\|\widetilde{X}\|\left\|\widetilde{X}^{-1 / 2} \Delta X \widetilde{X}^{-1 / 2}\right\| \\
=\theta_{2}\|\widetilde{X}\|\|R(\widetilde{X})\|=\theta\|R(\widetilde{X})\| .
\end{gathered}
$$

\section{Numerical Examples}

To illustrate the results of the previous sections, in this section, two simple examples are given, which were carried out using MATLAB 7.1. For the stopping criterion we take $\varepsilon_{k+1}(X)=\left\|X_{k}-\sum_{i=1}^{m} A_{i}^{*} X_{k}^{\delta_{i}} A_{i}-Q\right\|<1.0 e-10$.

Example 1. In this example, we study the following matrix equation:

$$
X-A_{1}^{*} X^{-1} A_{1}-A_{2}^{*} X^{-0.5} A_{2}=I,
$$

with

$$
\begin{gathered}
A_{k}=\frac{1 /(k+2)+2 \times 10^{-2}}{\|A\|} A, \quad k=1,2, \\
A=\left(\begin{array}{lllll}
2 & 1 & 0 & 0 & 0 \\
1 & 2 & 1 & 0 & 0 \\
0 & 1 & 2 & 1 & 0 \\
0 & 0 & 1 & 2 & 1 \\
0 & 0 & 0 & 1 & 2
\end{array}\right) .
\end{gathered}
$$

Algorithm (7) needs 10 iterations to obtain the unique positive definite solution

$$
X=\left(\begin{array}{ccccc}
1.0654 & 0.0506 & 0.0110 & -0.0008 & -0.0000 \\
0.0506 & 1.0764 & 0.0498 & 0.0110 & -0.0008 \\
0.0110 & 0.0498 & 1.0764 & 0.0498 & 0.0110 \\
-0.0008 & 0.0110 & 0.0498 & 1.0764 & 0.0506 \\
-0.0000 & -0.0008 & 0.0110 & 0.0506 & 1.0654
\end{array}\right)
$$

with the residual $\left\|X-A_{1}^{*} X^{-1} A_{1}-A_{2}^{*} X^{-0.5} A_{2}-I\right\|=5.0832 e-$ 011.

Example 2. In this example, we consider the corresponding perturbation bound for the solution $X$ in Theorem 10. We consider the following matrix equation:

$$
X-A_{1}^{*} X^{-1} A_{1}-A_{2}^{*} X^{-0.5} A_{2}=I,
$$

with

$$
\begin{aligned}
& A_{1}=\frac{1 / 3+2 \times 10^{-2}}{\|A\|} A \\
& A_{2}=\frac{1 / 6+3 \times 10^{-2}}{\|A\|} A, \\
& A=\left(\begin{array}{lllll}
2 & 1 & 0 & 0 & 0 \\
1 & 2 & 1 & 0 & 0 \\
0 & 1 & 2 & 1 & 0 \\
0 & 0 & 1 & 2 & 1 \\
0 & 0 & 0 & 1 & 2
\end{array}\right) .
\end{aligned}
$$

Choose $\widetilde{X}_{0}=A$. Let the approximate solution $\widetilde{X}_{k}$ of $X$ be given with the iterative method (7), where $k$ is the iterative number.

The residual $R\left(\widetilde{X}_{k}\right) \equiv I+A_{1}^{*} \widetilde{X}_{k}^{-0.5} A_{1}+A_{2}^{*} \widetilde{X}_{k}^{-0.25} A_{2}-\widetilde{X}_{k}$ satisfies the conditions in Theorem 10. By Theorem 10, we can compute residual bounds for $\widetilde{X}_{k}$ as

$$
\left\|\widetilde{X}_{k}-X\right\| \leq \theta\left\|R\left(\widetilde{X}_{k}\right)\right\|,
$$

where

$$
\begin{gathered}
\theta=\frac{2\left\|\widetilde{X}_{k}\right\|\left\|\widetilde{X}_{k}^{-1}\right\|}{\theta_{1}+\sqrt{\theta_{1}^{2}-4\left\|\widetilde{X}_{k}^{-1}\right\|\left\|R\left(\widetilde{X}_{k}\right)\right\|}}, \\
\theta_{1} \equiv 1+\left\|\widetilde{X}_{k}^{-1}\right\|\left\|R\left(\widetilde{X}_{k}\right)\right\| \\
-\left(0.5\left\|\widetilde{X}_{k}^{-1 / 4} A_{1}{\widetilde{X_{k}}}^{-1 / 2}\right\|^{2}+0.25\left\|\widetilde{X}_{k}^{-1 / 8} A_{2}{\widetilde{X_{k}}}^{-1 / 2}\right\|^{2}\right) .
\end{gathered}
$$

Some results are listed in Table 1.

The results listed in Table 1 show that the residual bound given by Theorem 10 is fairly sharp.

\section{Conflict of Interests}

The authors declare that they have no competing interests.

\section{Authors' Contribution}

All the authors carried out the proof. All the authors conceived the study and participated in its design and coordination. All the authors read and approved the final paper.

\section{Acknowledgments}

The authors would like to express their gratitude to the referees for their fruitful comments, which have led to 
the present form of Theorems 5 and 6 . The work was supported in part by the National Nature Science Foundation of China (11201263), Natural Science Foundation of Shandong Province (ZR2012AQ004), and Independent Innovation Foundation of Shandong University (IIFSDU), China.

\section{References}

[1] J. Zabczyk, "Remarks on the control of discrete-time distributed parameter systems," SIAM Journal on Control and Optimization, vol. 12, pp. 721-735, 1974.

[2] W. N. Anderson, Jr., G. B. Kleindorfer, P. R. Kleindorfer, and M. B. Woodroofe, "Consistent estimates of the parameters of a linear system," Annals of Mathematical Statistics, vol. 40, pp. 2064-2075, 1969.

[3] R. S. Bucy, "A priori bounds for the Riccati equation," in Proceedings of the Sixth Berkeley Symposium on Mathematical Statistics and Probability (Univ. California, Berkeley, Calif., 1970/1971), Vol. III: Probability Theory, pp. 645-656, Univ. California Press, Berkeley, Calif, USA, 1972.

[4] D. V. Ouellette, "Schur complements and statistics," Linear Algebra and Its Applications, vol. 36, pp. 187-295, 1981.

[5] W. N. Anderson, Jr., T. D. Morley, and G. E. Trapp, "The cascade limit, the shorted operator and quadratic optimal control," in Linear Circuits, Systems and Signal Processing: Theory and Application (Phoenix, AZ, 1987), C. I. Byrnes, C. F. Martin, and R. E. Saeks, Eds., pp. 3-7, North-Holland, Amsterdam, The Netherlands, 1988.

[6] W. Pusz and S. L. Woronowicz, "Functional calculus for sesquilinear forms and the purification map," Reports on Mathematical Physics, vol. 8, no. 2, pp. 159-170, 1975.

[7] B. L. Buzbee, G. H. Golub, and C. W. Nielson, "On direct methods for solving Poisson's equations," SIAM Journal on Numerical Analysis, vol. 7, pp. 627-656, 1970.

[8] L. A. Sakhnovich, Interpolation Theory and Its Applications, vol. 428 of Mathematics and Its Applications, Kluwer Academic Publishers, Dordrecht, The Netherlands, 1997.

[9] X. Duan, Q. Wang, and A. Liao, "On the matrix equation arising in an interpolation problem," Linear and Multilinear Algebra, vol. 61, no. 9, pp. 1192-1205, 2013.

[10] J. Li, "Perturbation analysis of the nonlinear matrix equation $X-$ $\sum_{i=1}^{m} A_{i}^{*} X^{p_{i}} A_{i}=$ Q," Abstract and Applied Analysis, vol. 2013, Article ID 979832, 11 pages, 2013.

[11] J. C. Engwerda, A. C. M. Ran, and A. L. Rijkeboer, "Necessary and sufficient conditions for the existence of a positive definite solution of the matrix equation $X+A^{*} X^{-1} A=Q$," Linear Algebra and Its Applications, vol. 186, pp. 255-275, 1993.

[12] J. C. Engwerda, "On the existence of a positive definite solution of the matrix equation $X+A^{T} X^{-1} A=I$, Linear Algebra and Its Applications, vol. 194, pp. 91-108, 1993.

[13] C.-H. Guo and P. Lancaster, "Iterative solution of two matrix equations," Mathematics of Computation, vol. 68, no. 228, pp. 1589-1603, 1999.

[14] A. Ferrante and B. C. Levy, "Hermitian solutions of the equation $X=Q+N X^{-1} N^{*}$, Linear Algebra and Its Applications, vol. 247, pp. 359-373, 1996.

[15] V. I. Hasanov and I. G. Ivanov, "On two perturbation estimates of the extreme solutions to the equations $X \pm A^{*} X^{-1} A=Q$," Linear Algebra and Its Applications, vol. 413, no. 1, pp. 81-92, 2006.
[16] V. I. Hasanov, I. G. Ivanov, and F. Uhlig, "Improved perturbation estimates for the matrix equations $X \pm A^{*} X^{-1} A=Q$," Linear Algebra and Its Applications, vol. 379, pp. 113-135, 2004.

[17] J. Li and Y. H. Zhang, "The Hermitian positive definite solution and its perturbation analysis for the matrix equation $X-$ $A^{*} X^{-1} A=Q$," Mathematica Numerica Sinica, vol. 30, no. 2, pp. 129-142, 2008 (Chinese).

[18] S. Vaezzadeh, S. M. Vaezpour, R. Saadati, and C. Park, "The iterative methods for solving nonlinear matrix equation $X+$ $A^{*} X^{-1} A+B^{*} X^{-1} B=Q$," Advances in Difference Equations, vol. 2013, article 229, 10 pages, 2013.

[19] I. G. Ivanov, V. I. Hasanov, and B. V. Minchev, "On matrix equations $X \pm A^{*} X^{-2} A=I$," Linear Algebra and Its Applications, vol. 326, no. 1-3, pp. 27-44, 2001.

[20] Y.-H. Zhang, "On Hermitian positive definite solutions of matrix equation $X-A^{*} X^{-2} A=I$," Journal of Computational Mathematics, vol. 23, no. 4, pp. 408-418, 2005.

[21] X.-G. Liu and H. Gao, "On the positive definite solutions of the matrix equations $X^{s} \pm A^{T} X^{-t} A=I_{n}$," Linear Algebra and Its Applications, vol. 368, pp. 83-97, 2003.

[22] V. I. Hasanov, "Positive definite solutions of the matrix equations $X \pm A^{*} X^{-q} A=Q$," Linear Algebra and Its Applications, vol. 404, pp. 166-182, 2005.

[23] J. Li and Y. Zhang, "Perturbation analysis of the matrix equation $X-A^{*} X^{-p} A=Q$," Linear Algebra and Its Applications, vol. 431, no. 9, pp. 1489-1501, 2009.

[24] H. Xiao and J. T. Wang, "On the matrix equation $X-A^{*} X^{-p} A=$ $Q(p>1)$, , Chinese Journal of Engineering Mathematics, vol. 26, no. 2, pp. 305-309, 2009.

[25] J. Li, "Solutions and improved perturbation analysis for the matrix equation $X-A^{*} X^{-p} A=Q(p>0)$," Abstract and Applied Analysis, vol. 2013, Article ID 575964, 12 pages, 2013.

[26] X. Yin and L. Fang, "Perturbation analysis for the positive definite solution of the nonlinear matrix equation $X$ $\sum_{i=1}^{m} A_{i}^{*} X^{-1} A_{i}=$ Q," Journal of Applied Mathematics and Computing, vol. 43, no. 1-2, pp. 199-211, 2013.

[27] J. Li and Y. Zhang, "Sensitivity analysis of the matrix equation from interpolation problems," Journal of Applied Mathematics, vol. 2013, Article ID 518269, 8 pages, 2013.

[28] S. M. El-Sayed and A. C. M. Ran, "On an iteration method for solving a class of nonlinear matrix equations," SIAM Journal on Matrix Analysis and Applications, vol. 23, no. 3, pp. 632-645, 2001/02.

[29] A. C. M. Ran and M. C. B. Reurings, "On the nonlinear matrix equation $X+A^{*} \mathscr{F}(X) A=Q$ : solutions and perturbation theory," Linear Algebra and Its Applications, vol. 346, pp. 15-26, 2002.

[30] A. C. M. Ran and M. C. B. Reurings, "A fixed point theorem in partially ordered sets and some applications to matrix equations," Proceedings of the American Mathematical Society, vol. 132, no. 5, pp. 1435-1443, 2004.

[31] A. C. M. Ran, M. C. B. Reurings, and L. Rodman, "A perturbation analysis for nonlinear selfadjoint operator equations," SIAM Journal on Matrix Analysis and Applications, vol. 28, no. 1, pp. 89-104, 2006.

[32] M. C. B. Reurings, "Contractive maps on normed linear spaces and their applications to nonlinear matrix equations," Linear Algebra and Its Applications, vol. 418, no. 1, pp. 292-311, 2006.

[33] M. C. B. Reurings, Symmetric matrix equations [Ph.D. thesis], VU University, Amsterdam, The Netherlands, 2003. 
[34] D. Zhou, G. Chen, G. Wu, and X. Zhang, "On the nonlinear matrix equation $X^{s}+A^{*} F(X) A=Q$ with $s \geq 1$," Journal of Computational Mathematics, vol. 31, no. 2, pp. 209-220, 2013.

[35] X. Duan, A. Liao, and B. Tang, "On the nonlinear matrix equation $X-\sum_{i=1}^{m} A_{i}^{*} X^{\delta_{i}} A_{i}=Q$," Linear Algebra and Its Applications, vol. 429, no. 1, pp. 110-121, 2008.

[36] Y. Lim, "Solving the nonlinear matrix equation $X=Q+$ $\sum_{i=1}^{m} M_{i} X^{\delta_{i}} M_{i}^{*}$ via a contraction principle," Linear Algebra and Its Applications, vol. 430, no. 4, pp. 1380-1383, 2009.

[37] X. Shi, F. Liu, H. Umoh, and F. Gibson, "Two kinds of nonlinear matrix equations and their corresponding matrix sequences," Linear and Multilinear Algebra, vol. 52, no. 1, pp. 1-15, 2004.

[38] X.-F. Duan, Q.-W. Wang, and C.-M. Li, "Perturbation analysis for the positive definite solution of the nonlinear matrix equation $X-\sum_{i=1}^{m} A_{i}^{*} X^{\delta_{i}} A_{i}=$ Q," Journal of Applied Mathematics \& Informatics, vol. 30, no. 3-4, pp. 655-663, 2012.

[39] R. Bhatia, Matrix Analysis, vol. 169 of Graduate Texts in Mathematics, Springer, New York, NY, USA, 1997. 


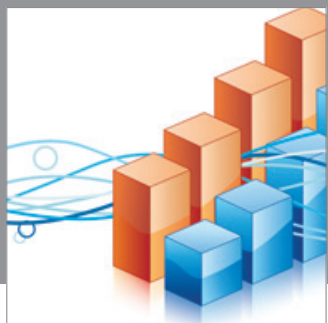

Advances in

Operations Research

mansans

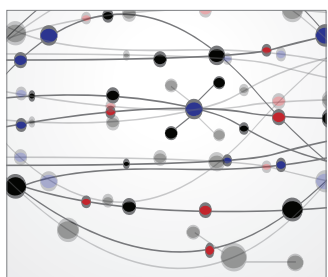

The Scientific World Journal
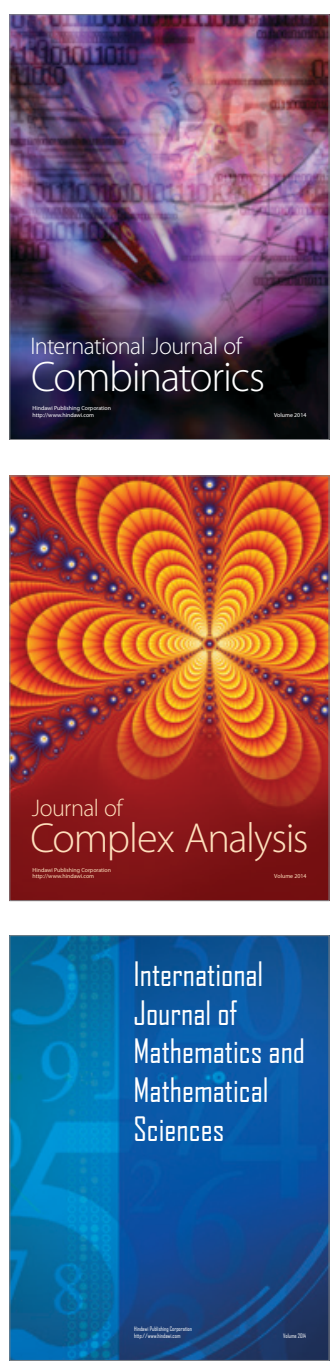
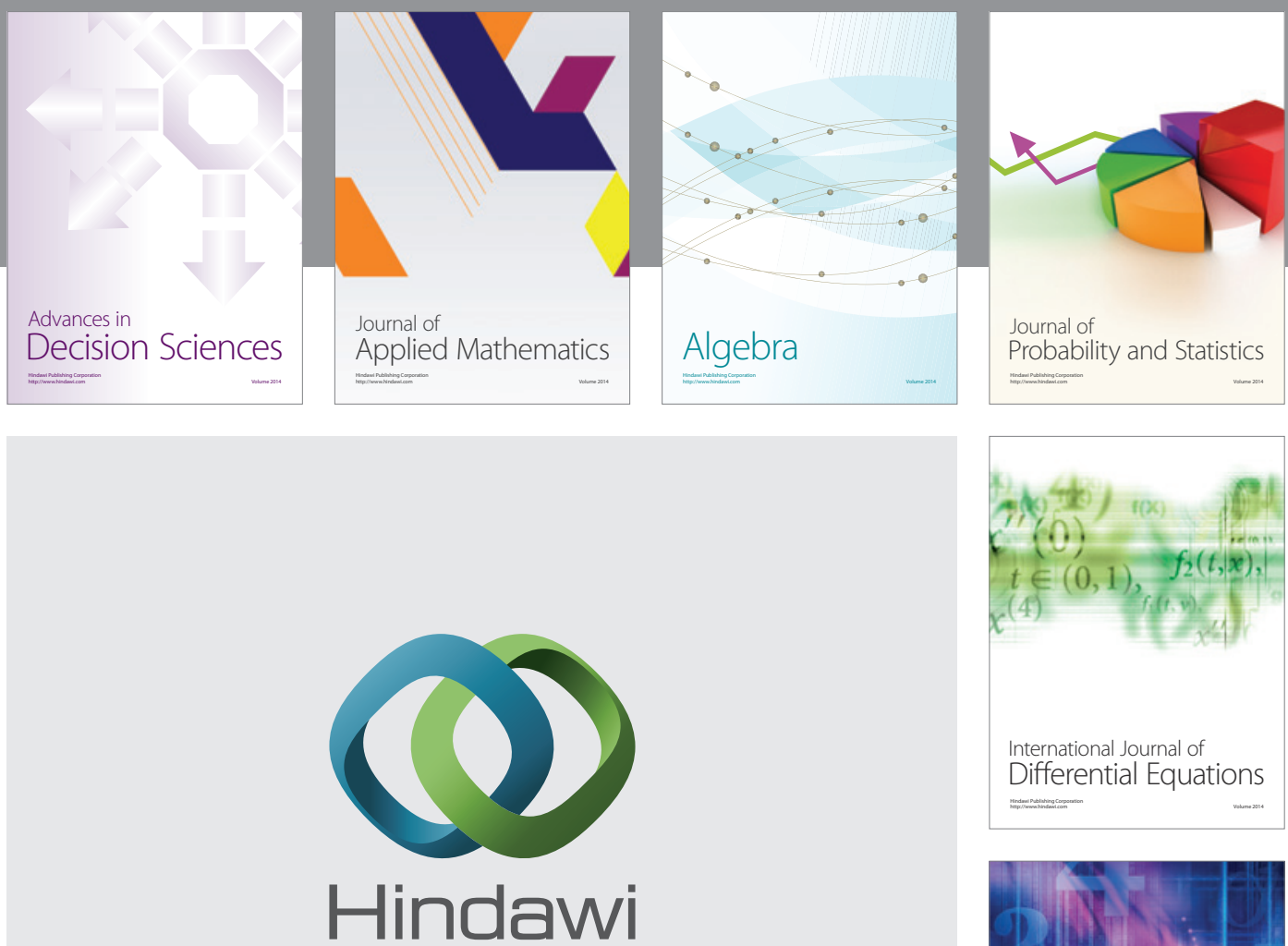

Submit your manuscripts at http://www.hindawi.com
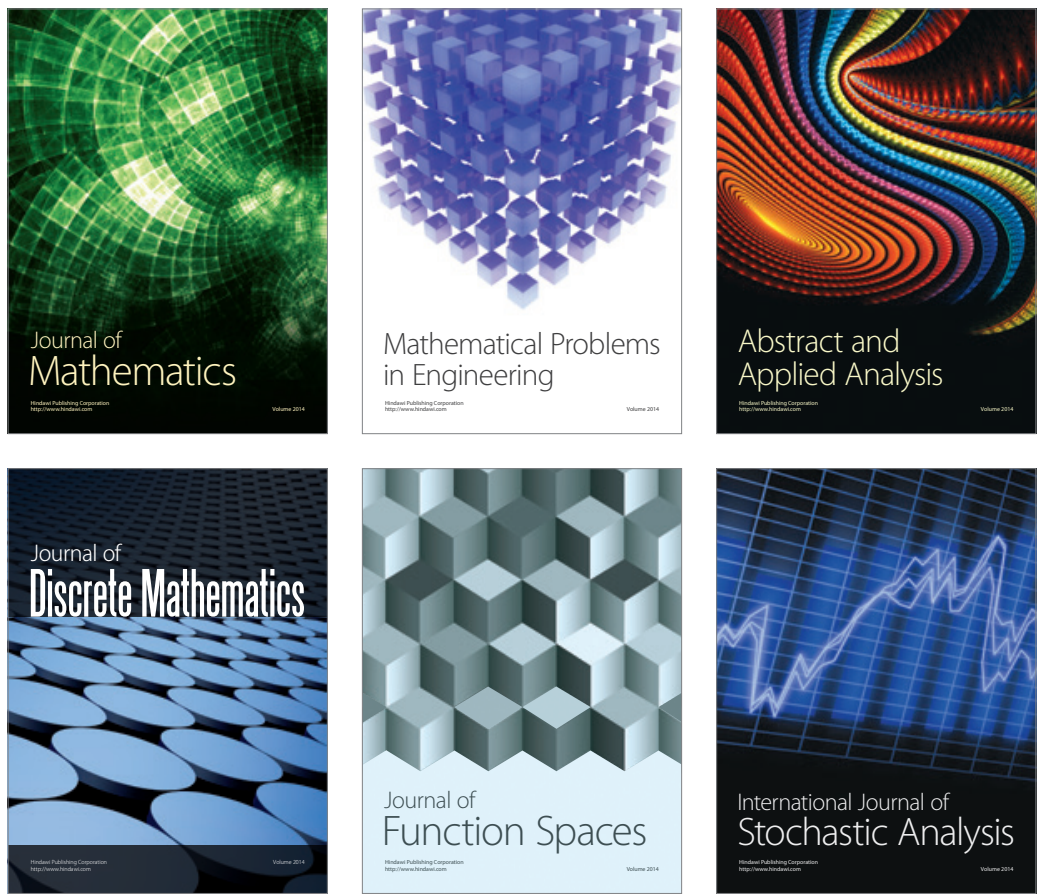

Journal of

Function Spaces

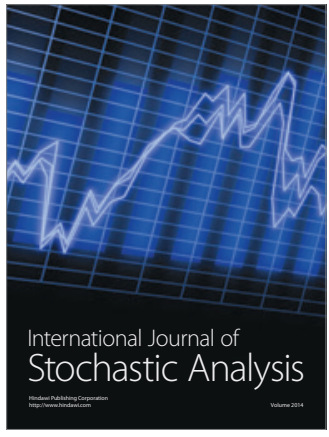

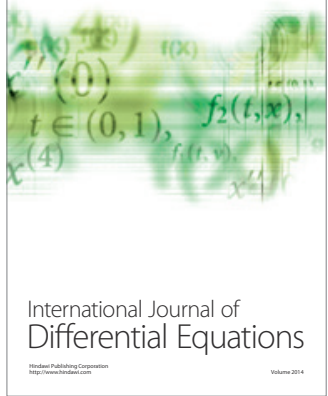
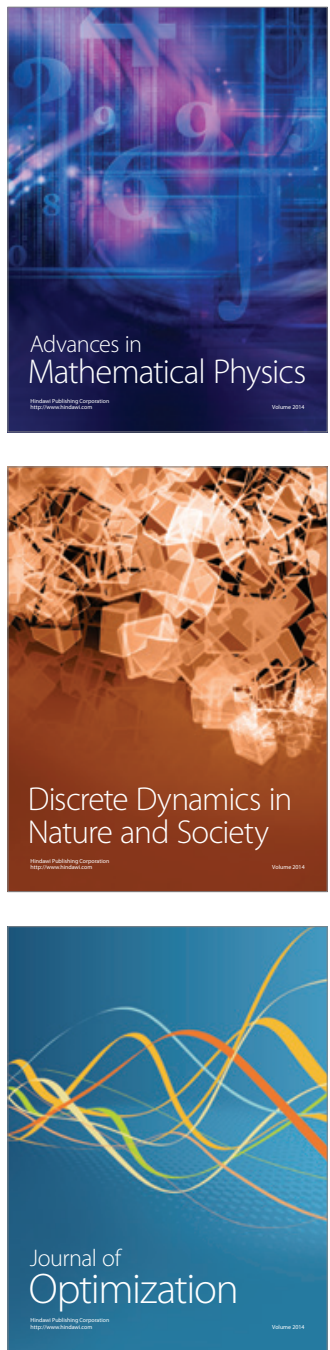